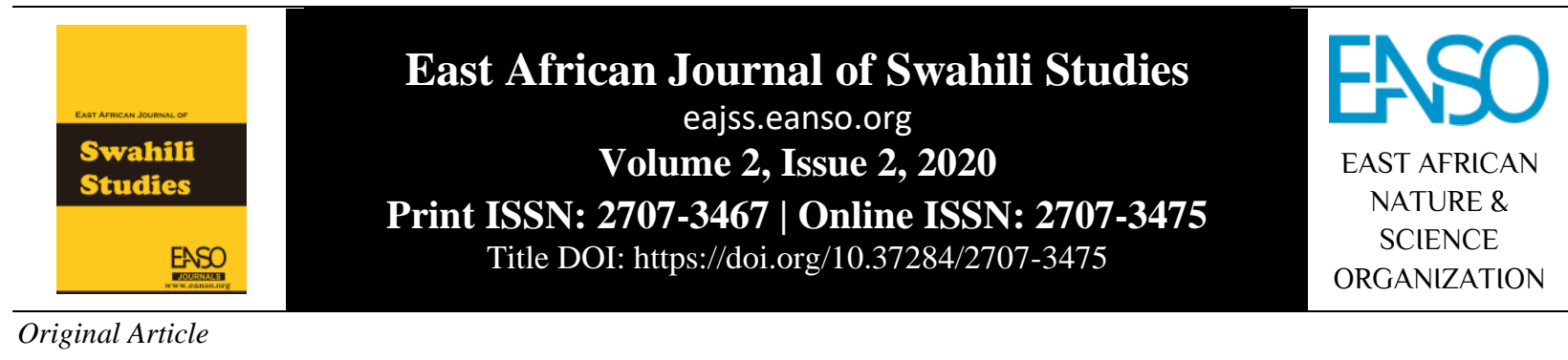

\title{
Mwingilianomatini katika Tamthilia ya Utangamano Wetu Ushindi Wetu (2017) ya Anitha E. Mgimba
}

\author{
Furaha J. Masatu ${ }^{*}$, Venancia F. Hyera ${ }^{1}$, Osmunda R. Ndunguru ${ }^{1}$ \& Aneth K. Ponera ${ }^{1}$ \\ ${ }^{1}$ Mwenge Catholic University, S.L.P. 1226, Moshi - Kilimanjaro, Tanzania. \\ *Barua pepe ya mawasiliano: mwanadiplo@yahoo.com
}

DOI ya Nakala: https://doi.org/10.37284/eajss.2.2.217

\section{Tarehe ya Uchapishaji: IKISIRI}

18 Septemba 2020

Istilahi Muhimu:

Mwingilianomatini,

Utangamano Wetu

Ushindi Wetu,

Anitha E. Mgimba,

Majazi,

Mwangwi wa Kifalsafa,

Anwani za Sura,

Mithiolojia ya Ishara.
Mwingilianomatini ni mbinu, stadi ama taaluma na mwongozo wa uchunguzi wa matini kisayansi, hususani kazi za kisanaa na zisizo za kisanaa ili kubaini kuwepo kwa athari ya uchopekaji ama usemezano; yaani kufanana kifani, kimaudhui na kimbinu kati ya matini moja na nyingine. Ufanano huo huweza kuwa wa moja kwa moja au kuwapo kwa ukaribiano. Mwingiliano huo hutambulika pia kama mwangwi. Inaaminika kwamba, mwingilianomatini ni miongoni mwa mbinu kongwe za kiutunzi, ilhali kiuhakiki inaonekana bado ngeni, haijashughulikiwa sana katika uga wa fasihi na sanaa ya Kiswahili. Kwa mantiki hiyo, makala haya yatasaidia kuongeza maandiko ya Kiswahili yaliyoshughulikia eneo la uhakiki kupitia nadharia ya mwingilianomatini ili kubaini mwingilianomatini uliopo katika matini, kwa mfano tamthilia ya Utangamano Wetu Ushindi Wetu. Matokeo ya uchambuzi yamebaini kuwapo kwa mwingilianomatini mwingi unaofungamana na historia ya makuzi na malezi ya mwandishi kidini (kiroho), kimwili (kiutamaduni na kimaadili), kijamii na kitaaluma - kwa namna moja ama nyingine. Sehemu ya matokeo hayo ndiyo inayobeba makala haya. Hivyo basi, makala yanawasilisha mwingilianomatini kati ya tamthilia teule ya Utangamano Wetu Ushindi Wetu na maandiko kutoka vitabu vitakatifu, majazi katika uteuzi na uumbaji wa wahusika, mwangwi wa kifalsafa, anwani za sura au ploti, na mithiolojia ya kiishara.
APA CITATION

Masatu, F., Hyera, V., Ndunguru, O., \& Ponera, A. (2020). Mwingilianomatini katika Tamthilia ya Utangamano Wetu Ushindi Wetu (2017) ya Anitha E. Mgimba. East African Journal of Swahili Studies, 2(2), 128-143. https://doi.org/10.37284/eajss.2.2.217 


\section{CHICAGO CITATION}

Masatu, Furaha, Venancia Hyera, Osmunda Ndunguru, and Aneth Ponera. 2020. "Mwingilianomatini katika Tamthilia ya Utangamano Wetu Ushindi Wetu (2017) ya Anitha E. Mgimba". East African Journal of Swahili Studies 2 (2), 128-143. https://doi.org/10.37284/eajss.2.2.217.

\section{HARVARD CITATION}

Masatu, F., Hyera, V., Ndunguru, O. and Ponera, A. (2020) "Mwingilianomatini katika Tamthilia ya Utangamano Wetu Ushindi Wetu (2017) ya Anitha E. Mgimba", East African Journal of Swahili Studies, 2(2), pp. 128-143. doi: 10.37284/eajss.2.2.217.

\section{IEEE CITATION}

F. Masatu, V. Hyera, O. Ndunguru, and A. Ponera, "Mwingilianomatini katika Tamthilia ya Utangamano Wetu Ushindi Wetu (2017) ya Anitha E. Mgimba”, EAJSS, vol. 2, no. 2, pp. 128-143, Sep. 2020.

\section{MLA CITATION}

Masatu, Furaha, Venancia Hyera, Osmunda Ndunguru, and Aneth Ponera. "Mwingilianomatini katika Tamthilia ya Utangamano Wetu Ushindi Wetu (2017) ya Anitha E. Mgimba”. East African Journal of Swahili Studies, Vol. 2, no. 2, Sept. 2020, pp. 128143, doi:10.37284/eajss.2.2.217.

\section{UTANGULIZI}

Uchambuzi ni mchakato wa kisayansi wa kuyatalii matini kwa lengo la kubaini kilichomo ndani, sababu ya kuwamo hicho kilichobainika, namna kitu hicho kilivyoumbwa na maana inayopatikana katika matini husika kwa lengo la kutoa ufafanuzi kulingana na hadidu za rejea za mchambuzi. Makala haya yanawasilisha uchambuzi uliolenga kubaini kuwapo kwa mwingilianomatini katika tamthilia ya Utangamano Wetu Ushindi Wetu ya Anitha E. Mgimba. Je upo? Na kama upo je, ni wa namna gani? Na kwa kiwango gani? Aidha, uchambuzi umeonesha dhahiri kuwapo kwa mwingilianomatini mkubwa ndani ya tamthilia hii. Hata hivyo, makala haya yanawasilisha mambo matano pekee yaliyomakinikiwa katika tamthilia teule. Mambo hayo ni matini za kidini (maandiko kutoka vitabu vitakatifu), majazi katika uteuzi na uumbaji wa wahusika, mwangwi wa kifalsafa, anwani za sura au ploti, na mithiolojia ya ishara.

\section{KIHUNZI CHA NADHARIA}

Uchambuzi uliongozwa na nadharia ya Mwingilianomatini. Nadharia hii ilichipuka kutoka katika mawazo ya usemezano ya mwanazuoni wa Kirusi, Mikhail Bakhtin (kuz. 1895, kuf. 1975) kisha yakaboreshwa na Mwanasemiotiki wa Kifaransa, Julia Kristeva (Wamitila, 2002; Wafula na Njogu, 2007). Hapa ni sawa na kusema kuwa, Julia Kristeva ndiye mwasisi wa nadharia ya Mwingilianomatini baada ya kuiboresha nadharia ya Usemezano ya Mikhail Bakhtin - katika miaka ya 1960s. Bakhtin hutuaminisha kwamba, kazi yoyote ile ya fasihi huwa na sauti tofauti tofauti zinazoingiliana na kupiga mwangwi kutoka kazi bunilizi zilizotangulia kuumbwa (Mningo, 2015). Kwa hiyo, kila kazi inayofuatia lazima itakuwa na mwangwi wa kazi ya awali. Kutoka katika mawazo ya Bakhtin, Kristeva akaibua misingi kadhaa iliyosababisha kuzaliwa kwa nadharia ya Mwingilianomatini (Wamitila, 2002 \& 2003; Wafula na Njogu, 2007).

Misingi hiyo ni (i) siyo rahisi na haiwezekani kuiangalia matini kama zao ambalo ni yatima, katika upekee wake na kwa kujibagua, badala yake ni lazima tutambue kuwa kuna matini tangulizi yenye kuhusiana na matini mpya; (ii) matini huingiliana na kuchagizana, kwa hiyo kufikiria ya kwamba matini ya kifasihi inajitosheleza na kujitegemea ni kujitia upofu; na (iii) matini ya kisanaa hukaribisha mitindo fulani ya kiusemi na misimbo fulani fulani ambayo husaidia kuashiria jambo, tukio, kazi au kazi nyingine - ijayo; hivyo kuleta athari kwa kazi bunilizi ijayo (Kristeva, 1980). Kutokana na misingi hii, tunaweza kusema, mwingilianomatini ni nadharia na stadi za kitaaluma zinazochunguza, kutambua na kufafanua mahusiano yaliyopo kati ya matini moja na matini nyingine, ili kubaini athari za ama moja kwa moja au kimwangwi zinazotokana na matini hizo kuingiliana na kukamilishana. Kwa maneno mengine, nadharia ya mwingilianomatini ni mwongozo wa uchunguzi wa kazi za sanaa, kisayansi hususani maandiko ya kisanaa ili kubaini kuwepo kwa athari ya uchopekaji ama usemezano; yaani kufanana kifani, kimaudhui na kimbinu kati ya matini moja na matini nyingine. Kufanana huko ni kule kwa moja kwa moja au kuwapo kwa 
ukaribiano. Mwingiliano huu kwa maneno mengine huitwa mwangwi.

Jambo la muhimu kwa msomaji wa makala haya ni kutambua ya kwamba, mwingilianomatini hauwezi kutambulika katika matini kama mchambuzi hana usuli wala ufahamu juu ya maarifa, mbinu, stadi na tabia zilizomo katika matini mbalimbali tangulizi. Kwa hiyo, mtu anayeweza kubaini mwingilianomatini katika kazi za kisanaa, hana budi kuwa mjuzi na mchokonozi wa kiwango cha juu, mwenye kiu na njaa ya maarifa, mbinu, stadi na tabia ya uchokonozi kupitia kazi bunilizi mbalimbali na maandiko ya taaluma anuai. Kinyume chake, msomaji hataweza kung'amua mwingilianomatini uliyomo katika kazi bunilizi anayoisoma kwa sababu hatasikia ndani ya ubongo wake mwangwi wa maarifa, mbinu, stadi na tabia zinazopatikana katika kazi tangulizi.

Sababu hii haitofautiani na kile kinachoelezwa kwa kina na Taylor na Taylor (1983) katika kitabu chao maarufu kiitwacho The Psychology of Reading. Katika kitabu hiki, wataalamu hawa wanaeleza kuwa, kwa kawaida maumbile ya ubongo wa mwanadamu huwa na sehemu yenye kurekodi, kuhifadhi na kurejesha kwa haraka sana kumbukumbu ya mambo na matukio yaliyowahi kumkuta mtu. Kwa hiyo, msomaji anapokutana na jambo au mbinu aliyowahi kukutana nayo katika kazi fulani aliyowahi kuisoma hapo awali; haraka sana, akili yake hutabasamu na kuamsha hisi za kuvuta urejesho wa jambo au mbinu husika. Urejeshi wa tukio, jambo au mbinu ya awali dhidi ya kile alichokutana nacho huzalisha makutano ya matini mbili. Makutano haya huzalisha mwangwi wa tukio, mbinu au jambo ndani ya akili ya msomaji. Mambo haya yote hufanyika kwa haraka sana. Kumbe basi, huwezi ukatambua kuwapo kwa mwingilianomatini kama wewe siyo msomaji makini au mfuatiliaji makini wa mambo na maandiko tofauti tofauti - kwa wingi na kwa kina.

\section{MUHTASARI WA TAMTHILIA YA UTANGAMANO WETU USHINDI WETU}

Utangamano Wetu Ushindi Wetu ni tamthilia ya Kiswahili yenye mwelekeo wa kifeministi zaidi inayosanisi athari za tabia na matendo ya baadhi ya wanajamii wanaoendekeza imani potofu, wivu, chuki, ubinafsi na manyanyaso kwa watu wanyonge; hasa wanawake na watoto. Tamthilia hii inatathmini athari hizo kisha kupendekeza namna ambavyo ukombozi wa kifikra, kiutamaduni na kijamii unavyopaswa kujenga umoja na ushirikiano kwa jamii kubwa ili kumsaidia mnyonge kujikomboa. Tamthilia imetumia lugha inayoeleweka kwa haraka na wepesi kwa mtu anayeimudu vema Lugha ya Kiswahili. Mijadala iliyojengwa katika tamthilia hii inaibua dhamira anuai kama vile ukombozi, nafasi ya mwanamke katika jamii, matabaka, hujuma, ubinafsi, fitina, dhuluma, chuki, wivu, tamaa, usaliti, unyonyaji, manyanyaso, ubaguzi, uwazi na ukweli, mila na desturi, umoja na mshikamano, malezi ya familia, juhudi na maendeleo endelevu, umuhimu wa kutunza nyaraka na utambuzi wa mali, usimamizi na uendelezaji mali, na kadhalika.

Tamthilia imesheheni majaribio na mbinu tofauti tofauti za kifani na kinadharia. Kwa mfano, ndani ya tamthilia mna mwingilianomatini kwa kila sura kupewa mada ndogo yenye kurejelea istilahi za kiisimu za taaluma ya isimu miundo ili kuonesha kuzaliwa hadi kukua kwa jambo linalojadiliwa na mwandishi. Mwingilianomatini mwingine umo katika matumizi ya michoro-picha, mbinu ya usimulizi na upanuzi usio wa kawaida wa miongozo ya jukwaa, matumizi ya ngoma, nyimbo, muziki, mashairi, majigambo, ngonjera, taswira ya Joka (Mgimba, 2017). Aidha, hata uteuzi wa jina "Sumukuvu" husadifu tabia na matendo yanayoakisi sifa za kitu au kiumbe husika kunaonesha sifa nyingine ya kazi kuwa na vionjo vya wahusika wa fasihi simulizi. Kwa mfano, mhusika Sumukuvu anaweza kutazamwa kama mhusika binadamu mwenye jina la aina ya sumu au kama mhusika wa fasihi simulizi aliyehaishwa kwa kupewa uhai kisha kutenda kama mwanadamu.

Katika tasnia ya Kilimo, Sumukuvu (mycotoxin) ni sumu au kemikali za sumu zinazotokana na aina ya fangasi wanaotambulika kwa jina la Kuvu ambao huota na kushambulia zaidi mazao ya chakula na mafuta yatokanayo na mimea itoayo majani na mbegu hasa jamii ya nafaka, mikunde, mizizi, karanga na malisho ya wanyama ndani ya shamba au ghala. Sumukuvu haionekani kwa macho bali kwa vipimo maalumu vya maabara. Zaidi ya hayo, tamthilia hii imezitambua taswira kadhaa zikiwemo joka, taa ya kijani, ukungu mzito, giza na ajali. Utajiri mwingine umo katika semi mbalimbali 
zikiwemo tamathali za usemi za aina tofauti tofauti, mathalani: chemsha bongo, nahau, vitendawili, methali na mafumbo. Vilevile, pamoja na majonzi, bado mwandishi ameingiza ucheshi kama njia ya kuleta faraja na amani iliyotoweka katika familia.

\section{KUHUSU MWANDISHI ANITHA ELIAS MGIMBA}

Anitha Elias Mgimba ni mtawa wa kike, Mtanzania, kutoka Shirika la Kikatoliki la Wamisionari wa Mt. Theresia wa Mtoto Yesu Iringa, Tanzania. Alijiunga na shirika hilo mnamo mwaka 2010 chini ya malezi ya Mama Mkuu wa wakati huo Sr. Teresina Gervasia Kindole. Anitha Elias Mgimba alizaliwa mnamo tarehe 24. 2. 1994 katika Kijiji cha Manga, Wilaya ya Ludewa, Mkoa wa Iringa (kwa wakati huo. Kwa sasa Wilaya ya Ludewa ni miongoni mwa wilaya zinazounda Mkoa wa Njombe) ambako ndiko wanakokaa wazazi wake hadi hivi leo. Anitha ni mtoto wa kwanza kati ya watoto sita kwa wazazi wakulima, Mzee Elias Mgimba na Mama Elimunda Mbigi. Watoto wengine na majukumu yao katika mabano ni Renhard Mgimba (Dereva), Johns Mgimba (Mwalimu), Rosamunda Mgimba (Nesi), Leticia Mgimba (Mwanafunzi, mafunzo ya umeme), na Mario Mgimba (Mwanafunzi, mafunzo ya uandishi wa habari). Elimu ya msingi aliipata katika Shule ya Msingi Manga (1999 - 2006) wakati elimu ya sekondari ya msingi (2007 - 2010) na elimu ya sekondari ya juu (2011 - 2013) alipata katika Shule ya Sekondari Pandahili, Mkoani Mbeya.

Elimu ya juu aliipata katika Chuo Kikuu cha Kikatoliki Mwenge, Moshi - Kilimanjaro (2014 2017) ambako alisoma na kuhitimu Shahada ya Kwanza ya Elimu katika Sanaa, akitaamilikia Historia na Kiswahili pasipo kusahau kozi ya Uandishi Bunilizi. Kwa sasa anajiendeleza kitaaluma - ngazi ya Shahada ya Uzamili ya Elimu katika Utawala na Mipango, Chuo Kikuu cha Mtakatifu Augustino Mwanza, Tanzania. Mbali na masomo ya uzamili, Anitha ni mwalimu wa sekondari akifundisha masomo ya Historia na Kiswahili katika shule ya shirika lake inayoitwa St. John Paul II Iringa. Chakula anachokipenda ni ugali kwa samaki. Pia, anapenda huduma ya ualimu kwa jamii. Anitha ni mchezaji mahiri wa mpira wa pete kupitia nafasi ya ufungaji - GK; nafasi anayoimudu vilivyo kiasi cha kumuongezea umaarufu, umakini na kujiamini kila aingiapo uwanjani kuikabili timu pinzani. Aidha, tofauti na watawa wengi, Anitha amepitia mafunzo ya Jeshi la Kujenga Taifa (J. K. T), Tanzania, kwa mujibu wa sheria katika Kambi ya $832 \mathrm{KJ}$ - J. K. T Ruvu, Mkoa wa Pwani, mnamo mwaka 2013. Sababu ya yeye kwenda jeshini ni Mama Mkuu (aliyechaguliwa kwa awamu nyingine - mara ya tatu), Sr. Teresina Gervasia Kindole, kuwahamasisha na kusisitiza kila aliyechaguliwa kujiunga na mafunzo hayo, kwenda mafunzoni bila kufikiria habari ya utawa wake. Uamuzi na msimamo huu wa Mama Mkuu unatokana na ukweli kwamba, hata yeye alipitia mafunzo ya J. K. T kwa mujibu wa sheria, hivyo haoni shida kwa watawa wake kuhudhuria mafunzo hayo, kutokana na faida alizozipata jeshini.

Tamthiliya ya Utangamano Wetu Ushindi Wetu ni kazi yake ya kwanza kuisana, kuichapisha na kuiingiza sokoni. Anitha anasema, katika maisha yake "hupendelea zaidi kusoma vitabu vya dini, sosholojia, lugha na fasihi hasa tamthilia kuliko aina nyingine za fasihi. Hata uandishi wa tamthilia hii una mbinu, na ulimwengu mpana zaidi kutoka katika matini bunilizi, matini ya kisosholojia, kifeministi na maisha halisi yanayotawala jamii zetu." Kumbe basi, tunaweza kusema ya kwamba, maisha ya utawa na maisha ya kawaida ya jamii inayomzunguka Anitha yamekutana katika umbo la kitamthilia na kusababisha kuzaliwa kwa tamthilia ya Utangamano Wetu Ushindi Wetu. Anitha ana ndoto ya kuandika kazi bunilizi nyingi na bora zaidi.

\section{MWINGILIANOMATINI KATIKA UTANGAMANO WETU USHINDI WETU}

Uchambuzi uliofanyika katika tamthilia hii umebaini kuwapo kwa mwingilianomatini mwingi katika tamthilia hii. Hata hivyo, kwa minajili ya makala haya, tutawasilisha mwingilianomatini unaohusiana na: maandiko kutoka vitabu vitakatifu, majazi katika uteuzi na uumbaji wa wahusika, mwangwi wa kifalsafa, anwani za sura au ploti, na mithiolojia ya ishara kama sehemu ya kutii matakwa ya idadi ya maneno iliyowekwa na jarida hili. 


\section{Maandiko kutoka Vitabu Vitakatifu}

Uchunguzi umeonesha ya kwamba, ndani ya tamthilia hii mna kauli zinazofanana au kukaribiana na nukuu kadha wa kadha katika maandiko matakatifu - Biblia Takatifu. Kubainika kwa nukuu au mwangwi huu kunatuthibitishia kuwapo kwa mwingilianomatini wa moja kwa moja au usiokuwa wa moja kwa moja ndani ya tamthilia ya Utangamano Wetu Ushindi Wetu. Jedwali lifuatalo linaonesha nukuu zinazopatikana ndani ya tamthilia husika na nukuu kutoka katika Biblia Takatifu zinazofanana au kukaribiana.

\section{Nukuu kutoka Utangamano Wetu Nukuu kutoka Biblia Takatifu Ushindi Wetu}
Naam mama'ngu. Ni tayari ndani Simulizi ya Safina ya Nuhu (Mwanzo sura ya 5 hadi ya 8). Mathalani, ya safina mama'ngu, twende. (uk. Mwanzo sura ya $7 ;{ }^{1}$ Bwana akamwambia Nuhu, ingia wewe na jamaa 3)
yako yote katika safina; [...]

Mungu atabaki kuwa Mungu. (uk. Zaburi 136:2 Mshukuruni MUNGU wa miungu; kwa maana fadhili zake 16) zadumu milele. Zaburi 118; ${ }^{5}$ Katika shida yangu nalimwita BWANA; BWANA akanijibu akaniweka panapo nafasi. ${ }^{6}$ BWANA yuko upande wangu, sitaogopa; mwanadamu atanitenda nini? [...] Zaburi $89{ }^{6}$ Maana ni nani katika mbingu awezaye kulinganishwa na BWANA? Ni nani afananaye na BWANA miongoni mwa malaika? [... ${ }^{8}$ BWANA, Mungu wa majeshi, Ni nani aliyehodari kama Wewe, Ee YAHU? [...]. Kumbukumbu la Torati 10:17 Maana Mwenyezi Mungu, Mungu wenu, ndiye Mungu wa miungu na Bwana wa mabwana. Yeye ni Mungu mkuu na mwenye nguvu, na wa kuogofya; asiyependelea nyuso za watu, wala hakubali rushwa. Ufunuo wa Yohana 19:16 Naye ana jina lake limeandikwa katika vazi lake na paja lake MFALME wa wafalme, BWANA wa mabwana [...]. Isaya 55:8 Maana mawazo yangu si mawazo yenu, wala njia zenu si njia zangu; asema BWANA.†

Yesu Kristu aliteswa na kufa kwa ajili yangu na ninyi wanangu. (uk. 16)

Isaya 53:3-6; ${ }^{3(a)}$ Alidharauliwa na kukataliwa na watu; [...] ${ }^{4(a)}$ Hakika ameyachukua masikitiko yetu, amejitwika huzuni zetu; [...] ${ }^{5}$ Bali alijeruhiwa kwa makosa yetu, Alichubuliwa kwa maovu yetu; Adhabu ya amani yetu ilikuwa juu yake, Na kwa kupigwa kwake sisi tumepona. Waebrania 2:3 Sisi je! Tutapataje kupona, tusipojali wokovu mkuu namna hii $?^{\dagger}[. .$.$] . Waefeso 1:7 Katika Yeye huyo, kwa damu yake, tunao$ ukombozi wetu, masamaha ya dhambi, sawasawa na wingi wa neema yake.

Yeye ndiye baba wa yatima na mume wa wajane. (uk. 16)

Na wewe amua kuwa, mme wako ni Yesu. (uk. 37)

Zaburi 68:5 Baba wa yatima na mwamuzi wa wajane, Mungu katika kao lake takatifu. Isaya 54: 4-8 ${ }^{4}$ Usiogope maana hutatahayarika; wala usifadhaike; maana hutaaibishwa; kwa kuwa utaisahau aibu ya ujana wako, pia mashutumu ya ujane wako hutayakumbuka tena. ${ }^{5} \mathrm{Kwa}$ sababu Muumba wako ni mume wako; BWANA wa majeshi ndilo jina lake; na Mtakatifu wa Israeli ndiye Mkombozi wako [...]. ${ }^{\dagger}$ Maana BWANA amekuita kama mke aliyeachwa na kuhuzunishwa rohoni, kama mke wakati wa ujana, atupwapo, asema Mungu wako.

Mungu atatulipia yote, nasi Mika 7:8 Usifurahi juu yangu, Ee adui yangu; niangukapo, nitasimama tutainuka tena. (uk. 16) tena; nikaapo gizani, BWANA atakuwa nuruni kwangu. ${ }^{\dagger}$ Isaya 14 (yote): ${ }^{27}$ Maana BWANA wa majeshi amekusudia, naye ni nani atakayelibatili? $\mathrm{Na}$ mkono wake ulionyoshwa, ni nani atakayeugeuza nyuma? ${ }^{29}$ Usifurahi, Ee Ufilisti, pia wote, kwa sababu fimbo ile iliyokupiga imevunjika; Maana katika shina la nyoka atatoka fira, Na uzao wake ni joka la moto arukaye ${ }^{\dagger 0}$ Nao walio masikini kabisa watakula, Na wahitaji 


\section{Nukuu kutoka Utangamano Wetu Nukuu kutoka Biblia Takatifu Ushindi Wetu}

\begin{tabular}{ll}
\hline & \\
& \\
& \\
& \\
Msihofu kamwe. \\
mnayoyaona leo hayana muda \\
mrefu kubaki historia. (uk. 16)
\end{tabular}

Kamwe dhiki zisitutenganishe wala kutufarakanisha na upendo wa Kristu Yesu [...]. (uk. 16)

Kila mmoja amshike sana Mungu na kumpenda mwenzake zaidi ya mwanzo. (uk. 16)

Siku akikanyaga... naomba mbingu iwe shahidi. (uk. 25)

Majaribu hayana budi kuja lakini ole wake ajaribuye. (uk. 28)

Kama BWANA aishivyo na Roho Yake iishivyo [...] (uk. 34)

[...] maana sijawahi kuona mwenye haki ameachwa. (uk. 34)

[...] Chonde chonde nakusihi kwa hayala na paa tafadhali sana tena sana $[. .$.$] (uk. 34)$

Shikeni sana elimu, msimuache aende zake maana huyo ndio uzima wenu. (uk. 36)

Mshikeni sana elimu msimuache awatoroke. (uk. 41) watajilaza salama; Nami nitatia shina lako kwa njaa, Na mabaki yako watauawa. Yoeli $2{ }^{25}$ Nami nitawarudishia hiyo miaka iliyoliwa na nzige, na parare, na madumadu, na tunutu, jeshi langu kubwa nililotuma kati yenu. ${ }^{26}$ Nanyi mtakula chakula tele na kushiba, na kulihimidi jina la BWANA, Mungu wenu, aliyewatendea mambo ya ajabu; na watu wangu hawatatahayari kamwe. ${ }^{\dagger}{ }^{27}$ Nanyi mtajua ya kuwa mimi ni katikati ya Israeli, na ya kuwa mimi ndimi BWANA, Mungu wenu, wala hakuna mwingine; na watu wangu hawatatahayari kamwe. ${ }^{\dagger}$

Kutoka 14:13-14: ${ }^{13}$ Musa akawaambia watu, Msiogope, simameni tu, mkauone wokovu wa BWANA atakaowafanyia leo; kwa maana hao Wamisri mliowaona leo hamtawaona tena milele ${ }^{\dagger 14}$ Bwana atawapigania ninyi, nanyi mtanyamaza kimya. ${ }^{\dagger}$

Warumi 8:35\&37: ${ }^{35} \mathrm{Ni}$ nani atakayetutenga na upendo wa Kristo? Je! Ni dhiki au shida, au adha, au njaa, au uchi, au hatari, au upanga? ${ }^{37}$ Lakini katika mambo haya yote tunashinda, na zaidi ya kushinda, kwa yeye aliyetupenda. ${ }^{\dagger}$

Mathayo Mtakatifu 22:37-39: ${ }^{37}$ Akawaambia, Mpende Bwana Mungu wako kwa moyo wako wote, na kwa roho yako yote, na kwa akili zako zote. ${ }^{\dagger}$ ${ }^{38} \mathrm{Hii}$ ndiyo amri kuu, tena ni ya kwanza. ${ }^{39} \mathrm{Na}$ ya pili yafanana nayo, nayo ni hii, Mpende jirani yako kama nafasi yako. ${ }^{\dagger}$

Kumbukumbu la Torati 30:19 Nazishuhudiza mbingu na nchi juи yenu hivi leo, kuwa nimeweka mbele yako uzima na mauti, Baraka na laana; basi chagua uzima, ili uwe hai, wewe na uzao wako.

Luka 17:1 Akawaambia wanafunzi wake, Makwazo hayana budi kuja, lakini ole wake mtu yule ambaye yaja kwa sababu yake!

2Wafalme 2:3, 4, 6 [Elisha] akasema, Kama BWANA aishivyo, na roho yake iishivyo, sitakuacha, [...]. 2Wafalme 4:30 Na mama yake yule mtoto akasema, Kama BWANA aishivyo, na roho yake iishivyo, sitakuacha. [...]. Yeremia 4:2 nawe utaapa hivi, Kama BWANA aishivyo, katika kweli, $[\ldots]$.

Zaburi 37:25-26: ${ }^{25}$ Nalikuwa kijana nami sasa ni mzee, lakini sijamwona mwenye haki ameachwa wala mzao wake akiomba chakula. ${ }^{26}$ Mchana kutwa hufadhili na kukopesha, na mzao wake hubarikiwa.

Wimbo Ulio Bora 3:5 Nawasihi, enyi binti za Yerusalemu, Kwa paa na kwa ayala wa porini, Msiyachochee mapenzi, wala kuyaamsha, Hata yatakapoona vema yenyewe.

Mithali 4:13 Mkamate sana elimu, usimwache aende zake; Mshike, maana yeye ni uzima wako. 


\section{Nukuu kutoka Utangamano Wetu Nukuu kutoka Biblia Takatifu Ushindi Wetu}

\begin{tabular}{ll}
\hline Niwaguse kama Thomaso kwa Yesu & Yohana Mtakatifu 20:24-25: ${ }^{24}$ Walakini mmoja wa wale Thenashara, \\
Kristu tu jamani. (uk. 40) & Tomaso, aitwaye Pacha, hakuwako pamoja nao alipokuja Yesu. ${ }^{25}$ Basi \\
& wanafunzi wengine wakamwambia, Tumemuona Bwana. Akawaambia, \\
& Mimi nisipoziona mikononi mwake kovu za misumari, na kutia kidole \\
& changu katika mahali pa misumari, na kutia mkono wangu katika ubavu \\
& wake, mimi sisadiki hata kidogo. ${ }^{\dagger}$
\end{tabular}

Utukumbuke katika sala zako ili Kumbukumbu la Torati 28:13 BWANA atakufanya kuwa kichwa, wala tukawe vichwa badala ya mikia. si mkia; nawe utakuwa juu tu, wala huwi chini; utakapoyasikiliza maagizo (uk. 41) ya BWANA, Mungu wako, nikuagizayo hivi leo, kuyaangalia na kufanya.

Jedwali hili linaonesha nukuu tulizozitambua kutoka tamthilia ya Utangamano Wetu Ushindi Wetu zenye kufanana au kushabihiana na nukuu kutoka katika Biblia Takatifu. Huu ni ushahidi usio na shaka kuwa, tamthilia hii haijatungwa kutoka hewani bali mwandishi amechota maarifa, stadi na tajiriba kutoka maandiko matakatifu ya dini iliyomlea na kumkuza, kama tulivyodokeza katika wasifu wa mwandishi. Nukuu zote tulizozirejelea, zinabeba kwa wazi mwangwi wa maandiko tangulizi hivyo kuthibitisha kwa dhati ya kwamba, utunzi na uandishi hauwezi kujitenga na athari ya matini tangulizi au mazingira yaliyomlea na kumkuza mwandishi - kimwili na kiroho. Ndio maana Worton na Still (1990) wanasisitiza kuwa, kila matini ni kijalizo cha matini nyingine. Hakuna matini iliyokamilika isiyohitaji kujalizwa na matini nyingine ili iweze kujitosheleza kimaana na kiuelewekaji. Hii ina maana kwamba, hakuna matini inayofanya kazi kama mfumo funge unaojitegemea wenyewe bali kila matini ni tegemezi kwa matini nyingine tangulizi. Aidha, athari hii ya mwingilianomatini wa kidini kutokana na kujifunza, kuyaishi maisha ya mafundisho ya dini na kusoma vitabu vitakatifu haupo kwa Anitha pekee, bali hata kazi bunilizi za watunzi nguli kama vile Abdilatif Abdallah, Euphrase Kezilahabi, Mwanakupona Binti Mshamu, Sayyid Abdalla Bin Ali bin Nasir, Shaaban Robert, Sheikh Umar bin alAhdaly, kwa uchache kutaja. Mtu ambaye siyo msomaji wa maandiko matakatifu hawezi kutambua makutano ya tamthilia hii na maandiko matakatifu mathalani Biblia Takatifu kwa sababu hana mifano tangulizi inayoweza kuamsha hisi zake za utambuzi na kumbukizi kupitia mwangwi.
Huu ni ushahidi usiopingika kwamba, mawazo na misingi ya Mikhail Bakhtin na Julia Kristeva siyo ya kusadikika bali halisi yenye mashiko kisayansi kwa kuwa (i) siyo rahisi na haiwezekani kuiangalia matini kama zao ambalo ni peke, katika upekee wake na kwa kujibagua, badala yake ni lazima kutakuwa na matini tangulizi yenye kuhusiana na matini mpya; (ii) matini huingiliana na kuchagizana, kwa hiyo kufikiria kwamba matini ya kifasihi inajitosheleza na kujitegemea ni upotofu; (iii) matini ya kisanaa hukaribisha mitindo fulani ya kiusemi na misimbo fulani fulani ambayo husaidia kuashiria jambo, tukio, kazi au kazi nyingine ijayo.

\section{Majazi katika Uteuzi na Uumbaji wa Wahusika}

Majazi ni mbinu ya kisanaa anayoitumia mwandishi kuteua jina linalosadifu tabia, mienendo na tajiriba za mhusika katika kazi anayoisana. Kwa mfano, mwandishi anaweza kumuita mhusika wake "Hasidi." Ili kusadifisha jina hili, mwandishi hulazimika kumjenga mhusika huyo kutenda mambo kwa husda mwanzo mwisho. Uteuzi na uumbaji wa tabia za wahusika unaolandana na majina yao ndiyo mbinu ya kisanaa inayoitwa majazi. Kwa kawaida, wahusika hawa hubeba tabia za wahusika bapa (wasiobadilika kitabia) ili kuyatendea haki majina yao kwani wakibadilika kitabia, basi majina hayatasadifu tabia mpya waliyoivaa. Kwa hiyo, wahusika hawa kitabia na kimsimamo huwa hawabadiliki tangu mwanzo hadi mwisho wa mchezo. Mlacha na Madumulla (1991) wanawaelezea wahusika wa namna hii kama wahusika ambao huwa hawaonekani kukua kitabia, kimatendo na kisaikolojia. Lengo kuu la mbinu hii ni kuushawishi umma kupenda matendo mema na 
majina yenye kubeba wema ili kuchukia uovu na kupinga matumizi ya majina yanayobeba ubaya kwani jamii za Kiafrika huamini kuwa jina hubeba uumbaji wa tabia husika. Ndio maana Waswahili husema, 'majina huumba.' Kupitia utangulizi wa tamthilia ya Utangamano Wetu Ushindi Wetu mwandishi Anitha E. Mgimba (uk. iii - iv), anakiri kutumia majazi kuumba mmoja wa wahusika wake. Tunanukuu:

[...] uteuzi wa jina "Sumukuvu" kusadifu tabia na matendo yanayoakisi sifa za kitu au kiumbe husika kunaonesha sifa nyingine ya kazi kuwa na vionjo vya wahusika wa fasihi simulizi. Kwa mfano, mhusika Sumukuvu anaweza kutazamwa kama mhusika binadamu mwenye jina la aina ya sumu au kama mhusika wa fasihi simulizi aliyehaishwa kwa kupewa uhai kisha kutenda kama mwanadamu. Katika tasnia ya Kilimo, sumukuvu (mycotoxin) ni sumu au kemikali za sumu inayotokana na aina ya fangasi wanaotambulika kwa jina la Kuvu ambao huota na kushambulia zaidi mazao ya chakula na mafuta yatokanayo na mimea itoayo majani na mbegu hasa jamii ya nafaka, mikunde, mizizi, karanga na malisho ya wanyama ndani ya shamba au ghala. Sumukuvu haionekani kwa macho bali kwa vipimo maalumu vya maabara. Viashiria ya kuwapo kwa sumukuvu katika zao ni pamoja na zao (mbegu) husika kuonekana kuoza au kuwa na rangi tofauti na ile ya asili yake.

Nukuu hii inathibitisha kuwapo kwa mwingilianomatini kati ya tamthilia ya Utangamano Wetu Ushindi Wetu na stadi za taaluma ya Kilimo. Lakini pia, huu ni ushahidi kwamba kuna usemezano wa kimbinu kati ya tamthilia ya Utangamano Wetu Ushindi Wetu na uteuzi na uumbaji wa wahusika katika kazi bunilizi za watunzi wengine akiwemo Shaaban Robert kupitia riwaya za Kufikirika, Utubora Mkulima na Kusadikika. Mbinu hii ya kuwapatia wahusika majina yanayoendana na tabia na matendo yao ni maarufu sana katika kazi bunilizi nyingi za Shaaban Robert (Ambrose, 2014; Saleh, 2015). Mathalani, katika riwaya ya Kusadikika kuna wahusika wawili wenye sifa za ubapa, ambao ni Karama na Majivuno. Karama ni mtu anayesawiriwa kuwa ni mtu mwema tu tangu mwanzo wa hadithi mpaka mwisho wa hadithi hiyo. Majivuno ni mhusika anayesawiriwa kama mtu mwenye majivuno na dharau kwa watu wengine tangu mwanzo mpaka mwisho wa hadithi ya Kusadikika (Ambrose, 2014). Mbali ya wema, mhusika Karama amechorwa kuwa mtu wa mapaji na matashi mema kwa jamii kupitia kusema na kuenenda kwake wakati Majivuno amekuwa ni mtu aliyejaa kibuli na kujivuna. Matendo ya Majivuno hayana tofauti na Matendo ya Sumukuvu katika tamthilia ya Utangamano Wetu Ushindi Wetu kwani ameonekana kuwa sumu kali kwa familia ya mdogo wake (Damiano) na ukoo wake mwenyewe. Ufuatao ni uthibitisho wa madai haya:

Damiano: 'It's ok my love.' Hasira hasara. (kimya kinatanda. Anaonekana kutafakari kitu. Ghafla anakata ukimya) Unajua sijui shida yake ni nini hasa kwenye nyumba hii? Matatizo yake kwetu ni...! Ama hapendi maendeleo yangu haya madogo au...? Vipi ningekuwa tajiri mkubwa... Sijui angefanyaje? (Anatoboa siri) Wiki tatu zilizopita, nilionana naye kule mjini. Tulisalimiana vizuri kisha tukaagana. Wakati nampa mgongo akanitamkia "unajidai sana bwana mdogo..." halafu akaondoka wanguwangu! Sikufanya chochote na sikuelewa anamaanisha nini! (uk. 7-8).

Caroline anatikisa kichwa kuonesha kukubali. Mzee 2 anahitimisha kikao. Ndugu wanapeana mikono kuashiria kikao kimekwisha. Hakuna anayempa mkono Sumukuvu. Wanatawanyika kila mtu kwao wakiwa wamejaa tafakuri isiyo kifani. Caroline anaondoka na wanawe kuelekea mjini. Huko mjini la kuvunda halina ubani, Sumukuvu anaendelea kufanya kila aina ya vitimbi lakini Caroline anaonekana kuzidi kuimarika zaidi na zaidi. Kila kitimbi kinachofanywa na Sumukuvu kinagonga mwamba. Majirani, wafanyakazi na mwajiri wa marehemu Damiano wako bega kwa bega na Caroline na familia yake. Askari polisi wasio waaminifu kwa taifa wanatumiwa na Sumukuvu kumtisha Caroline lakini njama zao zinagonga mwamba. Sikio la kufa halisikii dawa, Sumukuvu anapanga tukio la ujambazi nyumbani kwa Caroline. Hakuna siri ya watu wawili: hatimaye siri inavuja na askari polisi 
waaminifu wanaweka mtego. Wanafanikiwa kuwaua majambazi sita kati ya nane. Jambazi mmoja anafanikiwa kutoroka. Yule wa pili anajikuta akiishia mikononi mwa askari polisi waaminifu baada ya kupigwa risasi mguuni. Miongoni mwa majambazi waliouawa ni askari polisi! Naye majeruhi ni askari polisi. Sumukuvu kusikia hivyo anaamua kutoroka na kutokomea kusikojulikana. (uk. 26).

Tumenukuu baadhi ya kauli zinazoonesha sehemu ndogo ya tabia, mienendo na matendo ya Sumukuvu na athari yake kwa familia ya mdogo wake, familia yake mwenyewe, pia ukoo. Nukuu hizi na nyingine ambazo hatujazinukuu zinaonesha namna jina Sumukuvu lilivyogeuka sumu kwelikweli hata kusababisha kifo cha Damiano, baadhi ya askari wasiokuwa waaminifu kwa familia, wateja wa Sumukuvu kujikuta wakipata hasara pamoja na kesi ya kujibu baada ya kukamatwa na vitu vya wizi walivyonunua kutoka kwa Sumukuvu. Huku Sumukuvu mwenyewe akijitenga na familia pia ukoo kwa kukimbilia kusikojulikana baada ya kushindwa kuelewana na ukoo na kusikia anasakwa na jeshi la polisi kwa kesi ya kupanga njama za kupora na kusudio la kuua. Kabla ya Sumukuvu kuingia katika maisha ya familia na ukoo, jamii hizi zilikuwa zimestawi katika kila uga wa maisha. Hakuna aliyewahi kufikiria kama kuna siku zitaishia kuoza, visasi na mafarakano. Lakini baada ya Sumukuvu kuingia, jamii kama familia na ukoo wenye siha, upendo, umoja na mshikamano ilijikuta ikisaga meno na kuishia kusambaratika. Tunapoyatazama matendo ya mhusika huyu na jina lake - Sumukuvu, tunaishia kukubaliana na mwandishi kwamba, jina la mhusika limesadifu matendo yake.

Mbinu hii ya uteuzi wa majina ya wahusika kwa kuzingatia tabia na mienendo yao, pia taaluma mahususi kama tulivyoona jina Sumukuvu katika taaluma ya kilimo kuwa ni aina ya sumu kali inayoshambulia mazao na kusababisha madhara makubwa kwa mazao husika, mkulima, mlaji na uchumi ni uthibitisho wa kwamba tamthilia ya Utangamano Wetu Ushindi Wetu inasemezana na stadi za taaluma ya kilimo na mbinu ya majazi kama inavyojidhihirisha katika baadhi ya kazi bunilizi zikiwemo Kufikirika, Utubora Mkulima na Kusadikika - zote za Shaaban Robert. Makutano haya ya kitaaluma na kimbinu yanaongeza ushahidi wa utamu na utajiri wa kimatini unaopatikana katika tamthilia hii. Mintarafu ya maelezo hayo, ni dhahiri kwamba uchunguzi huu ulizingatia nadharia ya mwingilianomatini inayodai kuwa, mwingiliano wa kimatini katika utanzu wa kifasihi hujibainisha kwa njia ya urejeleaji wa vipengele vya matini tangulizi ndani ya matini mpya (Lemaster, 2012). Hivyo, urejeleaji au mwingiliano huu huisaidia hadhira kuibua maudhui yaliyokusudiwa na mwandishi wa matini husika.

\section{Mwangwi wa Kifalsafa na Kimtazamo}

Mwangwi ni sauti inayorejea kwa mara ya pili baada ya kutolewa kama vile mtu anapopiga kelele ndani ya pango au msituni, wakati falsafa ni elimu ya asili, maana na sababu za mambo au vitu, busara, hekima (Kamusi ya Kiswahili Sanifu, 2013). Kwa hiyo, mwangwi wa kifalsafa ni sauti inayorejea kwa mara ya pili baada ya kutolewa kupitia elimu ya asili, maana na sababu za mambo au vitu, busara na hekima. Tamthilia ya Utangamano Wetu Ushindi Wetu imempitisha mwanamke katika mapambano makali na machungu ya kujikomboa. Mchezo unaonesha namna mwanamke anavyojikuta akikata tamaa na kukubali kushindwa lakini kwa msaada wa jamii kubwa inayoungana naye, hatimaye anaibuka kidedea. Jambo hili ni mwangwi wa kifalsafa unaotukumbusha habari ya kidole kimoja kutokuvunja chawa na kwamba umoja ni ushindi daima. Falsafa hii ndiyo inayojitokeza katika tamthilia ya Nguzo Mama (2007) ya Penina Muhando. Tuanze kwa nukuu kutoka tamthilia ya Utangamano Wetu Ushindi Wetu kisha tutainasibisha na ushahidi kutoka tamthilia ya Nguzo Mama. Tunanukuu:

[...] Sumukuvu anaendelea kufanya kila aina ya vitimbi lakini Caroline anaonekana kuzidi kuimarika zaidi na zaidi. Kila kitimbi kinachofanywa na Sumukuvu kinagonga mwamba. Majirani, wafanyakazi na mwajiri wa marehemu Damiano wako bega kwa bega na Caroline na familia yake. Askari polisi wasio waaminifu kwa taifa wanatumiwa na Sumukuvu kumtisha Caroline lakini njama zao zinagonga mwamba. Sikio la kufa halisikii dawa, Sumukuvu anapanga tukio la ujambazi nyumbani kwa Caroline. Hakuna siri ya watu wawili: hatimaye siri inavuja na askari polisi waaminifu wanaweka mtego. [...]. Sumukuvu 
kusikia hivyo anaamua kutoroka na kutokomea kusikojulikana.

Caroline ana RB ya Polisi na amefanikiwa kukomboa baadhi ya mali za familia yake. Sasa amerudisha nyumbani gari moja na fedha zote zilizoko benki ambazo Jirani akishirikiana na bosi wa marehemu Damiano kwa msaada wa polisi waaminifu na mameneja wa mabenki husika walifanikiwa kuzifunga akaunti zote kwa kisingizio kuwa kadi na vitambulisho vya Damiano vilipotea katika eneo la ajali kwa sababu vilikuwa ndani ya waleti ambayo nayo haikuonekana. Mifugo nayo imepona baada ya Jirani kusema kuwa ni ya kwake na wako hapo kwa marehemu Damiano kwa kuwa kuna eneo la kutosha kuendesha ufugaji, pia ulinzi ni wa uhakika. [...] (uk. 25 - 26).

Caroline: Wee! Nahangaika kama kuku anayetaka kutaga mayai. Kidogo kidogo siku zinasonga mbele. $\mathrm{Na}$ mume wangu kabla hajatutoka alikuwa amenunua viwanja sehemu tofauti tofauti. Nashukuru Mungu fedha ya benki haikuguswa. Pia, mwajiri wake alitupatia ushirikiano mkubwa sana katika kufuatilia mafao ya marehemu. Fedha zote hizo kwa pamoja zikaniwezesha kujenga vibanda vya hadhi kwenye viwanja viwili ambavyo matunda yake yananipa kipato si haba. Kuna sehemu nyingine alikuwa akiongea kuwa "nitawapa ka-sapraizi kwenu. Mkipata nafasi mniambie." [...]. Kwa hiyo, huko kwenye hako kasapraizi ndo twalima na kuvuna chakula cha kutosha kuhifadhi mwaka mzima kwa ajili ya kula na kingine kuuza. (uk. 43)

Nukuu hizi zinaonesha juhudi za pamoja zilizotumika kumsaidia mwanamke kujikwamua kutoka katika makucha ya mwanaume dhalimu aliyejaa matashi ya mfumo dume - rohoni, hisiani na akilini mwake. Nukuu hizi zinaonesha mabadiliko ya kifikra ya jamii pana katika harakati za kumkomboa mwanamke kiuchumi, kijamii, kiutamaduni na kifikra. Kama siyo mchango wa Wazee, Majirani, Askari Polisi waaminifu, Vijana, Mameneja wa benki; Caroline asingelipata hali na mali alizorudishiwa hadi akafikia hatua ya kusema, "Shoga'ngu...! Mimi? [....] Aah! Nakwambia kwa mjomba sirudi tena! Hata sina habari zake. Nashukuru kwamba utangamano wetu umenipa ushindi dhidi ya hila za wakaanga sumu. Hakika kidole kimoja hakivunji chawa kamwe (uk. 44)." Maneno haya yanaaksi mwangwi wa mtazamo wa Penina Muhando kupitia tamthilia yake ya Nguzo Mama (1982/2007), kwamba, pasipo umoja na ushirikiano baina ya jinsi na jinsia zote, kamwe mwanamke peke yake hawezi kujikomboa wala kuuvunja mfumo dume. Ushahidi ni nukuu zifuatazo:

\section{BI MSIMULIZI:}

[...] Akina baba nao waliamka

Nyuma walifuatia na wao

Wajionee nini hicho

NGUZO MAMA.

Karibu walipofika wenyewe kujionea

Kwa marembo imeandikwa

Kubwa kubwa zake herufi

NGU - ZO MA - MA.

Ikatokea sauti

"Haya jamani Nguzo tusimamishe"

Haraka wakaivamia hao wabeba wana

Nguzo kutaka kusimamisha.

Lakini kina baba wa Patata

Njia wakageuza nyumbani

Kujirudia, vitandani kujilalia

Usingizi kujipigia

Kungojea jua litokee kisabeho kujipatia.

Moyoni walijiwazia wengine wazi

Walijisemea. Imeandikwa NGUZO MAMA

Hiyo NGUZO MAMA yao hao

Wabeba wana, waachieni wenyewe

Mambo wajitengenezee. (uk. 5-6)

[...] Wakainua, wakainua

Siku ya kwanza ikapita

NGUZO MAMA pale pale

Wakainua, wakainua

Usiku na mchana

Siku ya pili ikapita

NGUZO MAMA pale pale

Sasa leo ni siku ya tatu 
[Kwa hadhira] Niendelee, nisiendelee Niendelee, nisiendelee

[Kimya (uk. 55)

Tunaposoma tamthilia ya Nguzo Mama tunagundua ya kwamba, nguzo mama ilishindwa kusimama kwa sababu mmoja wa washikakamba hakuhusishwa wala hakutaka kuhusika katika wajibu wa kuhakikisha kuwa nguzo mama inasimama wima na kustawi. Mtu huyo siyo mwingine bali ni mwanaume - kama kundi maslahi. Mwanaume kama sehemu ya ukombozi wa mwanamke hapaswi kutengwa wala kujitenga na juhudi za dhati za ukombozi wa mwanamke. Hata kama wanaume wachache watapinga juhudi hizo, lakini kama kundi kubwa la wanaume watainuka kwa kumaanisha kwa dhati kumuunga mkono mwanamke, hakika "Nguzo Mama" itasimama hima kwani wanaume na wanawake wataungana kwa pamoja kusaidiana kuviondoa vikwazo vinavyokwamisha ukombozi wa kweli na endelevu wa mwanamke - kwa ustawi wa jamii nzima yenye kujaa usawa, umoja, upendo, haki, afya, uchumi, uhuru na mshikamano kwa maendeleo chanya na thabiti.

Kwa mantiki hii, maudhui yanayopatikana katika tamthilia ya Utangamano Wetu Ushindi Wetu yamerahisisha mno fasili ya tamthilia ya $\mathrm{Nguzo}$ Mama ambayo imechorwa kisitiara. Huku ndiko kusema, upo usemezano wa kifalsafa na kimtazamo kati ya Anitha E. Mgimba na Penina Muhando kupitia mjadala wa kutafuta suluhisho la njia bora ya kumkomboa mwanamke. Usemezano huo si mwingine bali falsafa ya kidole kimoja kamwe hakiwezi kuvunja chawa. Kwa maana kwamba, ukombozi wa kweli wa mwanamke hauwezi kuletwa kwa juhudi binafsi za mwanamke peke yake pasipo kushirikiana na mwanaume ili kuziondoa sirisila zinazomdhibiti mwanamke katika nyuga zote za maisha ya mwanadamu. Ndiyo maana Anitha amehitimisha mchezo wake kwa kusema, "[...]. Nakwambia kwa mjomba sirudi tena! [...]. Nashukuru kwamba utangamano wetu umenipa ushindi dhidi ya hila za wakaanga sumu. Hakika kidole kimoja hakivunji chawa kamwe."

\section{Anwani za Sura / Ploti}

Anwani za sura au ploti ni vichwa vya mada vinavyoteuliwa na mwandishi ili kutenganisha na kutambulisha tukio moja dhidi ya jingine. Anwani ndiyo inayobeba utambulisho wa sura au maonesho ndani ya tamthilia iliyo katika maandishi. Tamthilia ya Utangamano Wetu Ushindi Wetu inaonekana kugawa ploti zake kwa kutumia anwani mbili - kuu na ndogo. Anwani kuu (na anwani ndogo katika mabano) zilizotumika ni: Sehemu ya Kwanza (Neno), Sehemu ya Pili (Virai), Sehemu ya Tatu (Vishazi), Sehemu ya Nne (Sentensi), Sehemu ya Tano (Tungo), Sehemu ya Sita (Aya) na Sehemu ya Saba (Insha). Tunapotazama maneno yaliyotumika kama anwani ndogo za kutambulisha ploti, tunabaini maneno hayo yanatokana na taaluma ya Isimu - Mofolojia na Sintaksia kama yanavyotambulishwa na kujadiliwa katika Massamba, Kihore na Hokororo (2012) na katika taaluma za stadi za mawasiliano. Maneno haya si maneno tu bali dhana za kitaaluma zenye kurejelea vitu vya msingi katika taaluma hizi mbili. Kiisimu, tunatambua ya kwamba maneno haya ni istilahi ambazo hubeba maana za kitaaluma za neno husika ijapokuwa, mpaka sasa hakujawahi kupatikana muafaka wa maana au dhana hizi, "neno," "virai," "vishazi," "sentensi," "tungo," "aya" na "insha" (Kipacha, 2005). Pamoja na kutokuwapo kwa muafaka, hatuna budi kutambua ya kwamba upo ufafanuzi wa kitaaluma unaotolewa na wanataaluma wa stadi za Isimu. Kwa hiyo, kupitia ufafanuzi huo, tutaweza kuona kama uteuzi wa istilahi hizi za kiisimu unabeba mantiki ya mwingilianomatini kati ya kazi bunilizi ya kifasihi na taaluma ya Isimu.

Kamusi ya Kiswahili Sanifu (TUKI, 2013) inafasili neno "neno" kama mkusanyiko wa sauti zinazotamkwa au kuandikwa pamoja na kuleta maana. Na kwamba, maneno yanapowekwa katika makundi mbalimbali ambayo hayajafikia hadhi ya kishazi au sentensi huitwa Virai (Kipacha, 2005). Kwa hiyo, neno na neno ndiyo huunda virai. Navyo virai vinapowekwa pamoja huunda vishazi. Massamba (2004) anafafanua Kishazi kama "tungo yenye kiima na kiarifu ambayo imo ndani ya tungo kuu." Vishazi kadhaa vinapoungana huunda sentensi; nazo sentensi kadhaa zinapoletwa pamoja huunda tungo kisha tungo huunda Aya ambazo nazo huzalisha insha. Sentensi ni tungo inayojitosheleza ambayo kimsingi huhusisha tendo na mtendaji (TUKI, 2013). Kamusi hii ya Kiswahili Sanifu (TUKI, 2013) inafasili neno tungo ni kipashio cha kimuundo kinachotokana na uwekaji 
pamoja wa vipashio sahili ili kujenga kipashio kikubwa zaidi na Insha inaelezwa kuwa ni mtungo wa maneno kwa mtindo wa nathari juu ya jambo fulani. Kutokana na ufafanuzi huu tunagundua ya kwamba, neno (maneno) huzaa virai, navyo virai huzaa vishazi, navyo vishazi huzaa sentensi, nazo sentensi huzaa utungo, nazo tungo huzaa aya, kisha aya kadhaa huzaa insha. Kumbe basi, tunachogundua hapa ni kwamba mwandishi ameyapanga matukio yake kutoka tukio dogo kwenda tukio kubwa; yaani ameanza na jambo dogo lililozaa jambo kubwa, likazaa jambo kubwa kiasi, likazaa jambo kubwa kiasi zaidi, halafu likazaa jambo kubwa zaidi ya kiasi zaidi na hatimaye kukazaliwa jambo kubwa kuliko yote ambalo lilitokana na mkusanyiko wa mambo mengi. Huu ni mwingilianomatini kati ya fasihi na taaluma ya Isimu.

Si Isimu pekee inayoonekana kutoa mwangwi ndani ya tamthilia hii, bali pia upo mwangwi mwingine. Mwangwi huo ni kufanana kimbinu kwa Utangamano Wetu Ushindi Wetu na tamthilia kadhaa za Kiswahili, mfano Ngoswe Penzi Kitovu cha Uzembe (1988) ya Edwin Semzaba na Nje Ndani (2016) ya A. A. Majid Mswahili na M. S. Njonjolo. Edwin Semzaba ametumia stadi za taaluma ya Jiografia Maumbo kuteua na kutambulisha ploti ili kuonesha mzizi wa tatizo mpaka athari ya tatizo. Utambulisho huo umetumia dhana zifuatazo kimnyororo: Kijito - Vijito - Mto - Jito - Baharini. Nao A. A. Majid Mswahili na M. S. Njonjolo wametumia stadi za taaluma ya afya na tiba kuteua na kutambulisha ploti zao ili kudokeza mzizi wa tatizo mpaka athari ya tatizo. Wao wametumia mnyororo wa anwani ufuatao: Chachawizo - Onesho la Kwanza (Kipele) Onesho la Pili (Vipele) - Onesho la Tatu (Kijipu) Onesho la Nne (Jipu) - Onesho la Tano (Majipu) Onesho la Sita (Tambazi).

Kumbe basi, tamthilia ya Utangamano Wetu Ushindi Wetu ina athari ya stadi za mawasiliano na taaluma za Isimu - Mofolojia na Sintaksia katika uteuzi na utambulisho wa anwani za ploti zake. Lakini pia, tamthilia hii inasemezana kimbinu (uteuzi na utambulisho wa anwani za ploti) na tamthilia tangulizi kama vile Ngoswe Penzi Kitovu cha Uzembe na Nje - Ndani. Kufanana huku, na kuonekana kwa mwangwi wa taaluma za stadi za mawasiliano, Mofolojia na Sintaksia, pia mbinu ya anwani yenye kusadifu taaluma fulani kama tulivyoona katika tamthilia ya Ngoswe Penzi Kitovu cha Uzembe na ile ya Nje - Ndani, kunathibitisha mwingilianomatini usio na shaka katika uteuzi na utambulisho wa anwani za tamthilia ya Utangamano Wetu Ushindi Wetu na taaluma ya Isimu - Mofolojia, na kati ya tamthilia ya Utangamano Wetu Ushindi Wetu na tamthilia nyingine, mathalani Ngoswe Penzi Kitovu cha Uzembe na Nje - Ndani.

\section{Mithiolojia ya Kiishara}

Mithiolojia ya ishara ni mbinu ya kisanaa inayotumia taswira zinazotambulisha masimulizi jadia ya mwanadamu kuhusu mambo yanayofungamana na imani za nguvu asili na, au sihiri. Mwandishi ametumia ishara kadhaa zenye kufungamana na mithiolojia, hususani Mithiolojia za Kiafrika. Mojawapo ya ishara zenye kubeba mithiolojia maarufu miongoni mwa jamii nyingi za Kiafrika na kiulimwengu ni nyoka mkubwa mweusi (Joka). Kwa mujibu wa Morris (katika Shanafelt, 2014), nyoka kitaswira (hasa chatu) huhusishwa na mvua, maji, mnyororo wa ekolojia, uzazi na nguvu za sihiri/jadia, ishara ya mauti na ufufuko. Mithiolojia iliyobainishwa na mwanazuoni huyu juu ya nyoka na maana zake inaonekana kujitokeza pia katika maandiko matakatifu hususani Biblia Takatifu (Mwanzo 3:14-15, Mwanzo 44:5, Kutoka 7: 8-12, Hesabu 21:6-9, 1 Yohana 3:8, Ufunuo 12:9, Ufunuo 12:17, Ufunuo 20:2,10). Hii ndiyo sababu, nyoka kama taswira ana nguvu kubwa, kutokana na mapokeo ya kiimani katika tamaduni za jamii mbalimbali, si tu Afrika bali ulimwengu mzima (Hambly, 1929; Lynch na Roberts, 2010; Thomas, 2011; Shanafelt, 2014).

Maelezo haya yanatuongoza kutambua ya kwamba, matumizi ya joka hususani nyoka wanaotisha katika kazi bunilizi siyo kitu kipya bali ni mwendelezo wa matumizi ya sanaa kuchota tamaduni za kijamii mila, na kuziweka katika kazi bunilizi mpya. Katika tamthilia ya Utangamano Wetu Ushindi Wetu mwandishi anaonesha uhusiano kati ya ishara ya nyoka na maana yake kwa kuzingatia mithiolojia. Huku ndiko kusema, nyoka mkubwa ametumika kimithiolojia kutoa taarifa ya tukio lililombele ya jamii husika. Tunanukuu: 
[...] Anateka maji na kuondoka. Wakati anatembea ghafla anahisi nywele zake kumsisimka. Anasita kutembea. Anaangaza huku na kule, kule na huku, ghafla kwa mbele yake anamwona nyoka mkubwa mweusi anakatisha njia.

Caroline: (Hofu imemkamata, anapiga kelele) Mama we-e-e..., Nyoka-a-a-a. (Ndoo inaogopa na kuchupa kutoka kichwani. Caroline anapiga hatua kurudi nyuma kwa mwendo wa makitaimu). Mungu wangu nini hiki jamani! (Mwili umekufa ganzi kwa hofu. anasimama na kutikisa kichwa huku akisikitika na kujiongelesha) Kwa mila zetu ukimwona nyoka kama huyu lazima kuna kitu kibaya kitakachotokea katika familia au ukoo wa mtu huyo. Sijui...! Ni nini tena jamani? Tungoje tutasikia tu.

$$
[\ldots]
$$

Johns: Oooh! Leo na sisi wakati tunakwenda darasani tulimwona nyoka mweusi tititi anateremka mtini kutufuata. Tulipigaje kelele! Wanafunzi na walimu wakaja na mawe kumwua lakini alipotea sijui alikimbilia wapi mwenyewe. Mku-bwaaaa huyo!

Caroline: My God, what is that now! Ni nini hiki leo! Ilikuwa saa ngapi?

Tegemea: Yaani kama dakika moja hivi toka mlipotushusha na kuondoka.

Damiano: (Anawasoma kwa haraka na kutambua hisia zao) Msiwe na wasiwasi wowote, wapenzi wangu. Huyo ni kiumbe wa Mungu kama sisi. Kule porini wanafugwa na watu wanalipa hela nyingi kuwaona. Natambua Kiafrika, kwa baadhi ya jamii wakimwona nyoka mkubwa mweusi wanaona neema maishani mwao lakini wengine wanafikiria ni nuksi katika maisha yao. Mke wangu mpenzi, tafadhali usiwaze kabisa kama ni nuksi, zaidi waza kuwa kuna neema mbele yetu. Mimi ngoja nikachukue gari maana fundi kasema amekwisha kuikamilisha.

Caroline: (Kwa unyonge) Sawa darling ila uwahi kurudi.

Damiano: (Kwa tabasamu) Sasa hivi narudi my sweetie. M-mwa-a-a-a...

$$
[\ldots]
$$

\section{***Ukungu mzito unatanda***}

[...] Dakika 10 zimepita tangu Damiano aondoke pale nyumbani. Johns akiwa zizini anasikia kelele kwa mbali "u-uwi-wi-wi, ajali...! Ajali jamani! Jamani ajali njoo tusaidie-e-e-e." Anaangaza barabarani lakini haoni vizuri na hawezi kwenda huko bila idhini ya mama yake. Hivyo anakimbilia ndani kumtaarifu mama yake.

Johns: (Kwa sauti kubwa). Mama! Mama! Nasikia watu wanapiga kelele barabarani wanasema ajali! Ajali! Ajali!

Caroline: (Anasimama). Kuna aliyepona kweli? (Anajifunga kanga vizuri na kutoka ndani haraka). Hawa madereva wa mwendokasi doti komu watatumaliza, mwee!

Jirani: $\quad$ (Anakutana na Caroline nje ya geti na kumshika mkono). Mama Johns hebu rudi ndani kidogo. Kajiandae... ameshakimbizwa hospitalini... fanya haraka.

Caroline: (Anaonekana kuchanganyikiwa) Usiniambie ni Damiano wangu, eeh! Sivyo?

Jirani: (Hajibu. Anamshika Caroline na kumrudisha ndani kwa nguvu). 
Caroline: (Akionekana kuchanganyikiwa zaidi). Amekufa au kavunjika kidole? Niambie basi jamani au niache nikajionee mwenyewe. Jamani mume wangu mimi jamani...!

Jirani: (Hajibu. Bado amemshika Caroline kwa nguvu. Machozi yanamtiririka. Wamefika ndani. Anaonekana akifunga milango ya nje ya nyumba ya Damiano. Mara anaanza kupiga simu huku na kule. Wakati huo Caroline amekwisha kuingia chumbani huku akifuatiwa kwa nyuma na watoto wake).

Caroline: (Anatoka chumbani akiwa amevaa gauni refu. Kikoi kikiwa kimetupiwa kichwani badala ya begani. Hajielewi vizuri) Niko tayari jirani, twende. My blessed children, ngoja nikamuone baba yenu. Ile ajali ni yeye. Muwe na amani hajaumia sana. Nasikia kachubuka kaukucha tu (Watoto wanaitikia kwa wasiwasi hawana imani na maneno ya mama yao. Wanamgeukia Jirani ambaye amenyanyuka na kumsogelea Caroline).

Jirani: (Anamshika begani Caroline na kumuuma sikio) Hakuna haja ya kwenda hospitalini Mama Johns! Ajali ilikuwa mbaya sana. Lori limemsaga pa... (uk. 12-13)

Tumenukuu kwa urefu tukio hili ili kutoa picha kamili ya uhusiano wa kuonekana kwa nyoka mkubwa miongoni mwa wanafamilia wa Damiano na kilichojiri baadaye. Kumbe, kuibuka kwa nyoka huyu mweusi mkubwa katika familia ya Damiano kulikuwa ni taarifa kwa njia ya ishara ya mauti kama alivyosema Caroline, "[...] Kwa mila zetu ukimwona nyoka kama huyu lazima kuna kitu kibaya kitakachotokea katika familia au ukoo wa mtu huyo. Sijui...! Ni nini tena jamani? Tungoje tutasikia tu." Aidha, nyoka huyo aliashiria kuwapo kwa nguvu za sihiri zinazoweza kuleta madhara kwa familia au ukoo. Nguvu za sihiri kama alivyosema Morris (katika Shanafelt, 2014) zinaweza kutumia nyoka kufanyika ishara mojawapo ya kimithiolojia. Ushahidi wa nyoka kuonesha ishara ya kuwapo usihiri inapatikana kupitia nukuu hii:

[...] Vijana walioukimbiza mwili hospitali wanawasili. Taarifa yao inawakata maini waombolezaji. Damiano siyo mwili tena bali vipande vya nyama na mifupa [...]. Jeneza lililobeba mwili wa marehemu Damiano linaingizwa ndani ya nyumba aliyoijenga marehemu. Wazee wanaomba faragha kidogo ili wakamilishe taratibu za kimila kama ilivyo desturi yao. Baada ya dakika kadhaa wanatoka ndani huku mioyo yao ikivuja damu ishara ya kifo kisichokuwa cha kawaida kwa mujibu wa utamaduni wao. Rada zao zimeonesha kila kitu lakini hawataki kumwaga damu zaidi. Wanaagiza mwili utolewe nje na kuagwa haraka kabla ya jua halijawatoroka. (uk. 14$15)$.
Kupitia nukuu hizi tunabaini ya kwamba tamthilia ya Utangamano Wetu Ushindi Wetu imebeba mwingilianomatini wa kiishara wenye kuaksi mithiolojia jadia za Mwafrika na jamii nyingine nje ya bara la Afrika. Aidha, nyoka mkubwa kutumika kama ishara ya kisanaa hakuko katika tamthilia hii pekee bali pia katika kazi bunilizi nyingine za Kiswahili. Fasihi simulizi kama sanaa kongwe inaonekana kutumia sana nyoka wakubwa na viumbe wengine kumwakilisha Mungu, miungu, mizimu, binadamu, au dhana fulani kwa njia ya taswira. Kwa hiyo, taswira ya viumbe mathalani nyoka wakubwa ni sehemu ya utajiri unaobebwa katika masimulizi jadia tunayoyaona hata katika fasihi simulizi na fasihi andishi za kijadia (Bertoncini, 1989; Madumulla, 2009). Baadhi ya kazi bunilizi zenye usemezano wa nyoka mkubwa kimaana na kiishara, tunazoweza kuzihusisha na tamthilia ya Utangamano Wetu Ushindi Wetu ni pamoja na: Adili na Nduguze (Robert, 1952), Mtu Maarufu katika Historia ya Usukuma (Boaz, 1974), Ngao ya Jadi (Hussein, 1976), Shujaa Gishegu (Masatu, hakuna mwaka), Nondo Mla Watu, Nunda Mla Watu na Hadithi Nyingine (Hellier, 1964), Ngoma ya Ng'wanamalundi (Mbogo, 1988) na Gamba la Nyoka (Kezilahabi, 2006).

Katika kazi hizi zote, matumizi ya nyoka mkubwa yamebeba umaajabu wa kisihiri unaoashiria mojawapo kati ya utajiri, mauti, ulinzi na usalama, uponyaji na afya, sihiri, uzazi, udhalimu, mateso, mamlaka, kutaja kwa uchache. Kazi hizi ni kongwe kuliko tamthilia tuliyoichunguza. Imani yetu ni kwamba, kwa vile mwandishi alisoma kazi bunilizi za waandishi na mitindo tofauti tofauti katika kozi 
za fasihi (zikiwemo kozi za: nadharia za fasihi na uhakiki, uandishi bunilizi, fasihi andishi na fasihi simulizi), kuna uwezekano mkubwa kwamba alichota taswira ya Joka - kwa kujua au kutokujua. Uchotaji huu umeleta usemezano wa kiishara kati ya tamthilia teule na ontolojia ya Kiafrika na kazi nyingine bunilizi.

\section{HITIMISHO}

Tamthilia ya Utangamano Wetu Ushindi Wetu, ijapokuwa ni kazi bunilizi ya kwanza kwa mtunzi mchanga, lakini uchanga huo umeonekana hauko kwenye uteuzi na uumbaji wa sanaa kiusemezano. Hii ina maana kwamba, uchanga wa mwandishi huyu upo katika hoja ya jumla ya idadi ya kazi alizozisana lakini siyo katika hoja ya utundu wa usanaji wa kazi bunilizi. Matini tuliyoyatalii yameonesha ukomavu wa mwandishi katika matumizi ya mbinu na stadi za nadharia ya mwingilianomatini kwa kutumia matini nyingi zenye kusemezana kwa kiwango cha juu kutoka taaluma na sanaa tofauti tofauti.

\section{MAREJELEO}

Ambrose, B. M. (2014). Kuchunguza Masuala ya Kisiasa katika Riwaya za Shaaban Robert: Mfano wa Kusadikika na Kufikirika. Tasnifu ya Shahada ya Uzamili katika Kiswahili (Haijachapishwa). Chuo Kikuu Huria cha Tanzania.

Bertoncini, E. Z. (1989). Outline of Swahili literature. Leiden: E. J. Brill.

Bible Society of Tanzania. (1997). Maandiko Matakatifu ya Mungu Yaitwayo BIBLIA. Nairobi: Africa Area Typesetting Unit.

Boaz, B. Z. (1974). Mwanamalundi: Mtu Maarufu katika Historia ya Usukuma. Dar es salaam: Wizara ya Elimu ya Taifa.

Hambly, W. D. (1929). The Serpent in African Belief and Custom. In American Anthropologist, [N. S., 31].

Hellier, A. B. (1964). Nunda Mla Watu na Hadithi Nyingine. London: Sheldon Press.
Hussein, E. N. (1976). Jogoo Kijijini na Ngao ya Jadi. Dar es salaam: Oxford University Press.

Kezilahabi, E. (2006). Gamba la Nyoka. Nairobi: Vide Muwa Publishers.

Kipacha, A. (2005). Osw 101: Utangulizi wa Lugha na Isimu. Dar es Salaam: Chuo Kikuu Huria cha Tanzania.

Kristeva, J. (1980). Desire in Langauge: A Semiotic Approach to Literature and Art. New York: Columbia University Press.

Lemaster, T. (2012). What is "Intertextuality"? United States: University of WisconsinMadison.

Lynch, P. A. \& Roberts, J. (2010). African Mythology A to Z, (second edition). New York: Chelsea House Publishers. https://img.fireden.net/tg/image/1454/78/1454 787608049.pdf.

Madumulla, J. S. (2009). Riwaya ya Kiswahili: nadharia, historia na misingi ya uchambuzi. Dar es salaam: Mture Educational Publishers Ltd; Nairobi: Phoenix Publishers Ltd.

Masatu, F. J. (hakuna mwaka). Shujaa Gishegu na Hadithi Zingine.

Massamba, D. P. B. (2004). Kamusi ya Isimu na Falsafa ya Lugha. Dar es Salaam: TUKI.

Massamba, D. P. B., Kihore, Y. M., na Hokororo, J. I. (2012). Sarufi Maumbo ya Kiswahili Sanifu (SAMIKISA): Sekondari na Vyuo. Dar es Saalam: TUKI.

Mbogo, E. (1988). Ngoma ya Ng'wanamalundi. Dar es salaam: Education Services Centre Ltd.

Mgimba, A. E. (2017). Utangamano Wetu Ushindi Wetu. Dar - es - Salaam: E \& CE Publishers.

Mlacha, S. A. K. na Madumulla, J. S. (1991). Riwaya ya Kiswahili. Dar es Saalam: DUP.

Mningo, R. A. (2015). Tathmini ya Mwingilianomatini katika Utunzi wa Emmanuel Mbogo: Utafiti Linganishi wa Tamthiliya ya $\mathrm{Ngoma}$ ya $\mathrm{Ng}$ 'wanamalundi na 
Fumo Liongo. Tasnifu ya Shahada ya Uzamili katika Kiswahili (Haijachapishwa). Chuo Kikuu Huria cha Tanzania.

Mswahili, A. A. M. na Njonjolo, M. S. (2016). Nje - Ndani. Dar es salaam: Culture Link Africa Ltd.

Muhando, P. (2007). Nguzo Mama (Toleo la Pili). Dar es Saalam: Macmillan Aidan Ltd.

Robert, S. (1951). Kusadikika. London: Nelson.

Robert, S. (1952). Adili na Nduguze. London: Macmillan.

Robert, S. (1967). Kufikirika. Nairobi: OUP.

Robert, S. (1968). Utubora Mkulima. Nairobi: Nelson.

Saleh, K. K. (2015). Ufasihi Simulizi katika Riwaya za Shaaban Robert: Mifano kutoka Adili na Nduguze na Wasifu wa Siti Binti Saad. Tasnifu ya Shahada ya Uzamili katika Kiswahili (Haijachapishwa). Chuo Kikuu Huria cha Tanzania.

Semzaba, E. (1988). Ngoswe Penzi Kitovu Cha Uzembe. Dar es salaam: DUP

Shanafelt, R. (2014). Serpent Beings, Sacrificial Brides, Superboy Saviors: Comperative Analysis of African Serpent Lore. Katika Relegere: Studies in Religion and Reception, Juzuu I, Toleo 2.

Taylor, I na Taylor, M. M. (1983). The Psychology of Reading (toleo la kwanza). Cambridge, Massachusetts: Academic Press.

Thomas, R. (2011). Taswira ya Nyoka katika Kigano za Kisambaa. Tasnifu ya Shahada ya Uzamili katika Kiswahili (Haijachapishwa). Chuo Kikuu cha Dar es Salaam.

TUKI. (2013). Kamusi ya Kiswahili Sanifu (Toleo la 3). Dar es Salaam, Nairobi: Oxford University Press.

Wafula, R. M. na Njogu, K. (2007). Nadharia za Uhakiki wa Fasihi. Nairobi: Jomo Kenyatta Foundation.
Wamitila, K. W. (2002). Uhakiki wa Fasihi: Misingi na Vipengele Vyake. Nairobi: Phoenix Publishers.

Wamitila, K. W. (2003). Influence or Intertextuality? A Comparative Study of Kezilahabi's Nagona and Mzingile and Juan Rulfo's Pedro Paramo. Katika Kiswahili (Juzиu. 66). Dar es salaam: TUKI.

Wamitila, K. W. (2003). Kamusi ya Fasihi: istilahi na nadharia. Nairobi: Focus Publications Ltd.

Worton, M. na Still, J. (1990). Intertextuality: Theories and Practice. Manchester: Manchester University Press. 